\title{
Factors Affecting the Utilization of Sexually Transmitted Infections Health Services at the Primary Health Centers in EI-Damazin locality at Blue Nile State, Sudan 2015-2016
}

\author{
Khalid Fadl Alla Khalid ${ }^{1}$, Samia Yousif Idris Habani ${ }^{2}$, Nada Jafar Osman ${ }^{3}$, Malaz Elbashir Ahmed \\ ${ }^{1}$ HIV Prevention Program, United Nations Population Fund, Khartoum, Sudan \\ ${ }^{2}$ Free Lance Community Medicine Consultant, Khartoum, Sudan \\ ${ }^{3}$ Directorate General of Primary Health Care, Federal Ministry of Health, Khartoum, Sudan \\ Email Address: \\ elmalkey2011@gmail.com(K. F. A. Khalid),samiahabbani8@gmail.com (S. Y. I. Habani), nadajafar@gmail.com (N. J. Osman), \\ malazbashirahmed@hotmail.com (M. B. Ahmed)
}

\section{To cite this article:}

Khalid Fadl Alla Khalid, Samia Yousif Idris Habani, Nada Jafar Osman, Malaz Elbasir Ahmed. Factors Affecting the Utilization of Sexually Transmitted Infections Health Services at the Primary Health Centers in El-Damazin locality at Blue Nile State, Sudan 2015-2016. World Journal of Public Health. Vol. 3, No. 2, 2018, pp. 61-68. doi: 10.11648/j.wjph.20180302.15

Received: May 21, 2018; Accepted: June6, 2018; Published: July 12, 2018

\begin{abstract}
Sexually transmitted Infections (STIs) constitute a public health problem, especially in developing countries and among poor people, women and adolescent. In Sudan, despite the endorsement of STIs health services in 2004, within the primary health care (PHC) service package, the uptake of STIs by those in need remains limited. This study aimed at exploring the factors affecting the utilization of STIs health services at the PHC in El-Damazin locality in Blue Nile State (BNS) in Sudan, 2015 -2016. The Specific objectives of this study were: Assessing the capacity of PHC and care providers, in ElDamazin locality in (BNS), in providing quality STIs health services. The study was descriptive, analytical, cross-sectional health facility based study. The study investigated eight PHC and ten care providers during the study period. The study findings indicated that while $62.5 \%$ of the investigated PHC centers provide the STIs health services behind closed door, yet $75 \%$ of the investigated PHC centers do not abide by the necessary confidentiality measures in maintaining the records of the patients, as well as lacking some medical equipment. All the investigated eight PHCs centers lack management protocol of the STIs. Female health care providers (CPs) are only 30\%. While medical doctors represent $80 \%$ of the care providers, only $20 \%$ of them received training in STIs syndromic case management. Eighty percent of health CPs specified the lack of protocols and guidelines affects the quality of service to STIs patients. The study concluded that the lack of STIs management protocols and guidelines, lack of specific capacity in STIs syndromic case management, limited numbers of female care providers, insufficient medical equipment adversely affect the quality STIs services.
\end{abstract}

Keywords: Sexually Transmitted Infections, STIs Health Service Provision, STIs Syndromic Case Management, PHC Capacities, Care Providers' Capacities, Confidentiality of STIs Service Provision, Quality of STIs Service Provision

\section{Introduction}

STIs are the infection that transmitted from one person to another by sexual contact. STIs also referred to as sexually transmitted diseases (STDs) or venereal diseases (VDs). More than thirty pathogens including bacteria, viruses, and parasites are recognized causes of STIs. Some of them are curable and linked to the highest incidence of STIs, other are not curable [1].
WHO report in 2012, showed the distribution of the new curable STIs is different across regions in the world, and reflected the STIs is higher in developing regions (South-East Asia, where the figures showed one hundred forty-two million STIs cases in comparison to the eighteen million new cases in European region) [2]. The burden of morbidity and mortality worldwide resulting from STIs compromises the quality of life, as well as sexual and reproductive health and newborn and child health. STIs also facilitate the sexual transmission of HIV indirectly and cause cellular changes that precede 
some cancers. Moreover, STIs impose a substantial strain on the budgets of both households and national health systems in the middle- and low-income countries and hurt the overall well-being of individuals [3]. Currently, STIs constitute a public health problem especially in developing countries because they are frequent with high prevalence and incidence rates, they can cause an enormous burden of disease, especially among poor people, women, and children [4].

Few health facilities in the developing countries have laboratory equipment or skills for the etiological diagnosis. WHO developed and promoted the STIs syndromic case management approach in a large number of countries in the developing world as a tool to manage the STIs patients. The STIs syndromic case management approach is a flowchart or algorism used to identify a consistent groups of symptoms and easily recognized signs, and for the provision of treatment that will deal with the majority or most serious organisms responsible for producing a syndrome [4].

Concerning Sudan, the global AIDs response progress report $(2012$-2013) shows that the number of reported STIs cases has been steadily decreasing from 89,625 in 2011 , to 60,400 in 2012 , and 33,000 in 2013. The continuously declines attributed to the reporting weaknesses and nonsubmission of reports by high burden states in the past two years. Contrary to the previous figures, the surveillance rounds and blood bank screen data indicate increasing numbers of STIs such as syphilis, Hepatitis B, and C among the general population [5].

Sudan national surveys (1993-2000), indicated the majorities of STIs are acquired via heterosexual transmission, and showed the low level of knowledge among the general population on HIV/AIDS and STIs and the self-protection measures [6]. Sudan National AIDs Program (SNAP) database reports, indicated the number of STIs cases more 60,000 cases in 2012 . The prevalence rate of syphilis at $2.3 \%$ among pregnant women attending antenatal care services during 2010 [6]. The 2010- integrated Bio-Behavioral Surveillance survey (IBBS), shows that the prevalence rate of syphilis is $27.9 \%$ among most at risk women and $15 \%$ among most at risk men across all country with geographical variation [7] and the IBBS-2012 shows that the prevalence of abnormal vaginal discharge is $24.8 \%$, and the genital ulcer is $11.7 \%$ in BNS [8].

Sudan Federal Ministry of Health (FMOH) has adopted a district health system policy. This district health system policy introduced the PHC approach to healthcare provision at localities level at the all states in Sudan [3]. Principally the STIs services provides at the level of PHC as part of health services.

The management and responsibility for PHC decentralized to state and local levels, but the necessary funding transfers from the central government have remained insufficient and the health services have deteriorated over the past decade [5]. The district health system policy includes; health promotion, community participation, and the delivery of health services at local level by using the PHC system. Although Sudan health system treating the STIs patients by using the STIs syndromic case management approach in the PHC level since 2004, still the utilization of STIs health services remains limited. This low utilization might be force by many factors at demand side or at the supply side (e.g. lack of skilled staff, the care workers' attitude, late referral, the availability of treatment and lack of equipment) [9].

The National Reproductive health (NRH) strategy (2006 2010), realize that STIs are major public health problems, worsened by armed conflict and natural disasters [10]. The displacement of a considerable proportion of the population due to the civil war and unrest in BNS [11], augmenting the risk for acquiring STIs by a community. In BNs, the PHC provide STIs health services exists in two localities out of six localities (El-Damazin and Rosaries localities). The fifth population and household census -2008, reported a total population of 1,632,096 capita in the BNS. Out of this figure, 329,845 are male and female at the reproductive period (15 to 44 years). Based on the 2012 Antenatal care (ANC) Sentinel Sero- Survey, the estimation of STIs cases among this category of age around 3,298.45 cases (1\% prevalence rate among general) [12]. However, the number of cases registered by the BNS- MOH in 2012, indicated only $35 \%$ of the estimated STIs cases received STIs health services This means there are $65 \%$ of STIs cases not approaching the STIs health services, and this raising the questions regarding the barriers of not obtaining the STIs health services by the needy population, and this can be classified into barriers to access the STIs health services or barriers to receive appropriate STIs health services after reaching the PHC centers.

\section{Methods}

\subsection{Study Design}

This study is descriptive, analytical and cross-sectional health facility-based study.

\subsection{Study Area}

The study area was El-Damazin locality in BNS. The study area is defined based on the ratio of PHC centers in the localities at BNS. The number of PHC centers in BNS is 10, the PHC centers that provides STIs health services are available in two localities at BNS, namely in EL- Damazin, and Rosaries localities with a ratio of 8:2 respectively.

Background on the Study area: BNS is one of the eighteen states of the Republic of Sudan. It has an area of $45,844 \mathrm{~km}^{2}$ and an estimated population of 1,193,293. The State is subdivided into six localities, El-Damzin is one of them. ElDamazin city is the capital of El-Damazin locality as well as the capital of BNS. The region is host to around forty different ethnic groups. The state neighbors two countries; South Sudan and Ethiopia. Its economic activities are based on agriculture and livestock and increasing mineral exploitation. The health system in BNS is decentralized health system and the health services provided through one central hospital in El-Damazin city, rural hospital and the PHC centers at the localities level. 


\subsection{Study Population}

The study population refers to the community from which the sample population was selected, based on the inclusion criteria. In this research, the study population:

1) The PHCs centers in El-Damzin locality ( Namely; Elrabie' PHC, Police hospital PHC, Military hospital PHC, Hai Elzihoor PHC, EL-Damazin hospital PHC center, Ibn Hayan PHC center, Elshafie PHC center and Sheifa PHC center).

2) Health CPs' who provides the STIs health services at the PHC centers in El- Damazin locality.

\subsection{Sample Size}

The sample size included all PHC centers (Eight PHC canters) in El-Damzin locality, and all STIs care providers at the studied PHC centers (Eight medical doctors, one medical assistant, and one health visitor).

\subsection{Sampling Technique}

Total coverage sampling technique.

\subsection{Data Collection Tools}

\subsubsection{Check List to Study the PHC Centers}

This tool was used to observe the duration of service during the official working days, the privacy of the service provision, the availability of STIs management guidelines and protocols, the availability of information, educational and communication materials (IEC), the availability of STIs drugs and the availability of medical equipment requires for STIs service provision. Also, this tool used to obtain the sociodemographic data of STIs patients attended the PHC centers in the past three months prior to the study period from the PHC centers registries.

\subsubsection{Interviewer-Administered Questionnaires with Open and Close Ended Questions to Investigate the Health CPs at the PHC Centers}

This tool covered the sociodemographic data of CPs, the working experience years of CPs, wither they received inservice training on STIs or not, the challenges facing the CPs on STIs service provision, and the referral mechanism used by the CPs. Also, this tool included a pre designed STIs case studies to investigate the knowledge of health CPs on syndromic diagnosis of STIs.

\subsection{Data Collectors}

Five data collectors were nominated by the BN-SMOH. One of the five data collectors was the team supervisor.

The data collectors received three days training on the following; STIs, the study tools, the methodology of data collection and the ethical approach to study participants.

\subsection{Data Collection Methods}

Two data collection methods used in this study, the observational and direct interview method.

\subsubsection{Observing}

The data about the confidentiality of service provision, the equipment, drugs and records data in the studied PHC centers was obtained through observation, using check lists.

\subsubsection{Interviewing}

1: The data about the health CPs obtained by direct interview; the interviews were run directly by the supervisors of the teams of data collectors.

2: The knowledge of care providers' on syndromic diagnosis of STIs was measured by administered a pre designed STIs case problems with symptoms and signs and their answer were weighted based on syndromic diagnosis for each presented case.

\subsection{Data Management and Analysis}

\subsubsection{Data Management}

1: The validity of the questionnaires were tested practically in the PHC centers at Rosaries locality.

2: During the data collection period, the team supervisors supervised the data collection process to ensure that interviewing of the respondent was properly applied, and the data collectors followed the questionnaires and the check lists properly.

3: The raw data collected by data collectors, was subjected to regular examination in the meetings of data collectors. In these meetings, undiscovered errors were detected and cleaned manually.

\subsubsection{Data Analysis}

The data entered into Microsoft Excel office -2013. General analytical analysis to measures the frequency and percentage of trends. The analyzed data presented by cross tabulation, graphs.

\section{Results}

\subsection{Primary Health Care Centers Assessment Results}

The results showed, the eight PHC centers at El-Damazin locality in BNS provide the health services during the official working days from 9:00 AM to 16:00 PM ( $n=8)$.

Regarding the confidentiality of the services, figure one showed that $75 \%$ of the studied PHC centers had consultation rooms with working door, yet only $62.5 \%$ of the studied PHC centers provides the consultation with patients behind a closed door. Only two PHC keeping the patient's records' in place reachable only by care providers. 


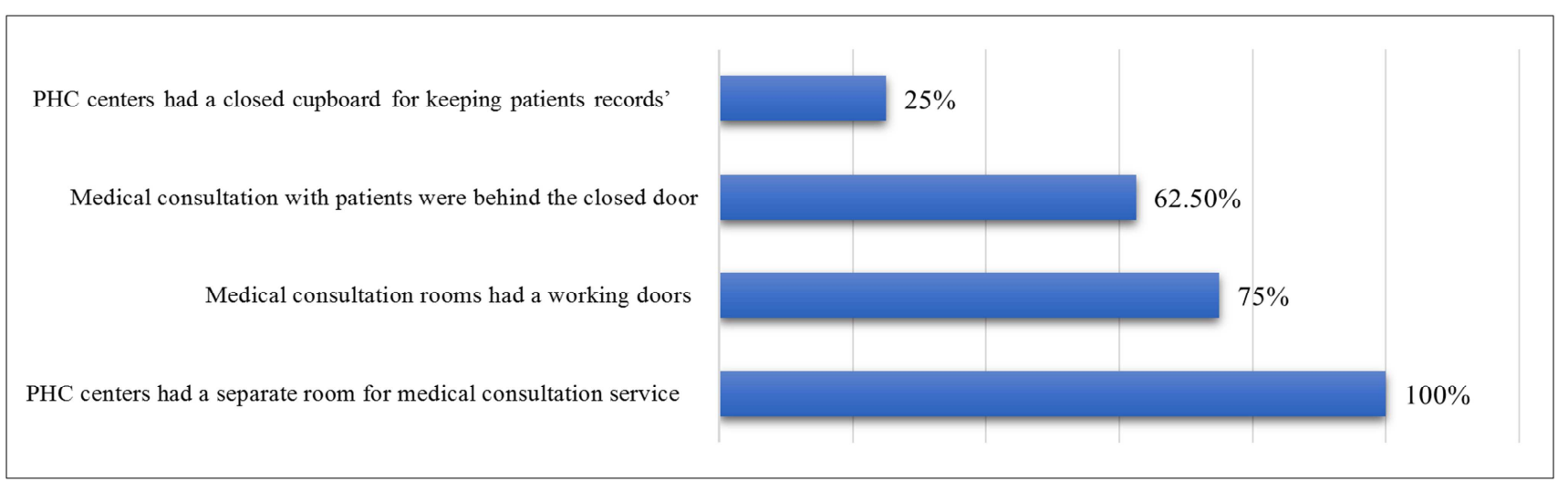

Figure 1. Confidentiality and the Availability of the privacy condition in the PHC centers under the study at El-Damazin locality in BNS, 2015 - 2016 ( $n=8$ ).

Figure two showed that the STIs management protocols not available in all PHC centers, IEC materials available only in $13 \%$ of the PHC centers, and seven out of eight PHC centers had a completed stock of STIs drugs.

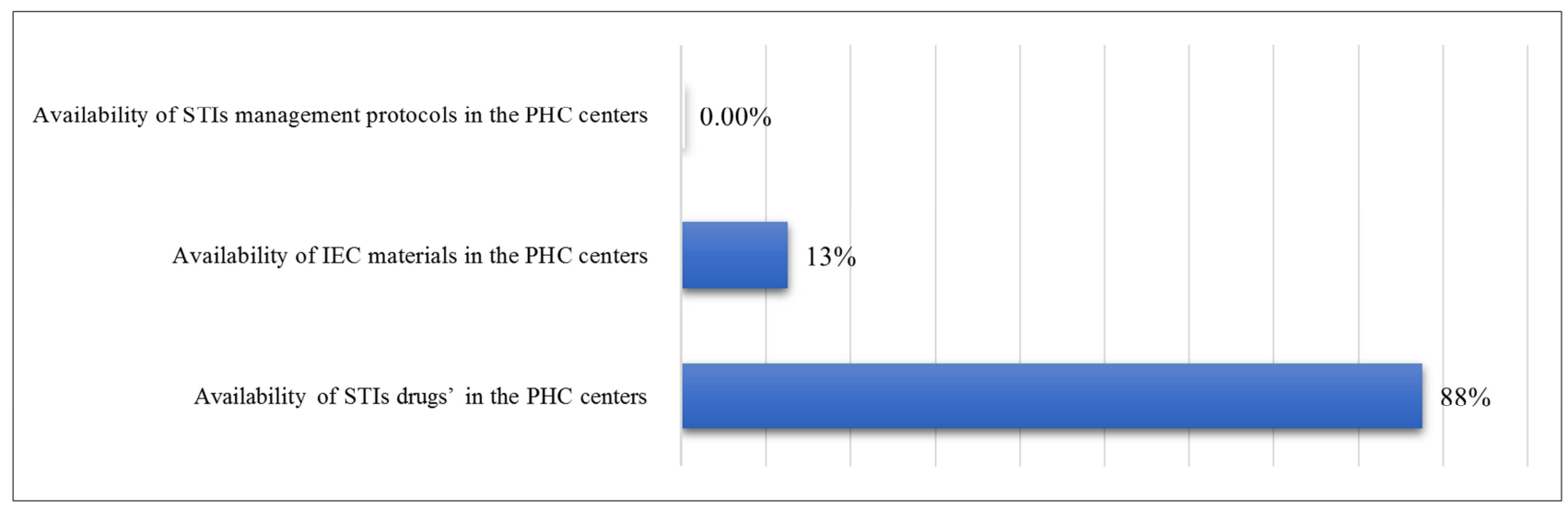

Figure 2. Proportion of PHC centers had STIs treatment protocol, stock of STIs drugs and STIS IEC materials to those did not at El-Damazin locality in BNS, $2015-2016(n=8)$.

Regarding the medical equipment, the results showed that only the medical gloves were available in all PHC canters, while cuscus instruments and the medical examination lamb were available in $25 \%$ of PHC centers. Condom not available in $50 \%$ of the studied PHCs

The study results showed that the overall ratio of STIs Diseases versus other diseases in the PHC centers records' at
El -Damzin locality was 1:4.3 (One STIs patients every five patients).

Table one showed that the overall ratio of STIs male patients to Female patients was 1:1.4 in the PHC registries during the three months prior to the study period. Also, the results showed that the higher frequency of STIs patients was among the age group of 25 to 34 years (53\%).

Table 1. Ratio of male STIs patients versus other female STIs patients in the PHC centers records' at El-Damzin locality in BNS during the last three months in 2015 prior to study $(n=7)$.

\begin{tabular}{llll}
\hline PHC & STIs male patient & STIs female patient & Ratio ( Male: female) \\
\hline Elrabie' & 8 & 4 & $1: 0.5$ \\
Police & 10 & 5 & $1: 0.5$ \\
Military & 20 & 50 & $1: 2.5$ \\
Hai Elzihoor & 30 & 15 & $1: 0.5$ \\
Damazin & 20 & 40 & $1: 2$ \\
Ibn Hayan & 5 & 15 & $1: 3$ \\
Shafie' & 5 & 10 & $1: 2$ \\
Total & 98 & 139 & $1: 1.4$ \\
\hline
\end{tabular}

\subsection{Health Care Providers' Assessment Results}

The study results showed that $70 \%$ of studied CPs at PHC centers in El-Damazin locality were male. Eighty percent of $\mathrm{CPs}$ are medical doctors. Seventy percent of CPs had working experience less than two years. Only $20 \%$ of health $\mathrm{CPs}$ received in - service training on STIs syndromic management approach and it was more than three years ago $(\mathrm{n}=10)$.

Regarding the knowledge of health CPs on diagnosis of STIs, Table two showed that the $70 \%$ to $60 \%$ of studied 
health CPs gave incorrect answer for first and second case studies respectively while $70 \%$ of them gave correct answer

for third case study $(n=10)$.

Table 2. The Knowledge on diagnosis of STIs patients among the care providers in the eight PHC centers at El-Damazin locality in BNS, 2015 - 2016 ( $n=10)$.

\begin{tabular}{|c|c|c|c|c|}
\hline \multirow[t]{2}{*}{ Care providers' answer } & \multicolumn{2}{|c|}{$\begin{array}{l}\text { Case study 1: A male patients' presented with penile } \\
\text { urethral discharge, burning micturition or frequent } \\
\text { urination }\end{array}$} & \multicolumn{2}{|c|}{$\begin{array}{l}\text { Case study 2: A female patient presented with vaginal } \\
\text { discharge, burning in micturition, itching or pain during sex }\end{array}$} \\
\hline & Freq. & $\%$ & Freq. & $\%$ \\
\hline Correct & 3 & 0.30 & 4 & 0.40 \\
\hline In correct & 7 & 0.70 & 6 & 0.60 \\
\hline Total & 10 & 100 & 10 & 100 \\
\hline
\end{tabular}

Table 2. Continued.

\begin{tabular}{lll}
\hline \multirow{2}{*}{ Care providers' answer } & \multicolumn{2}{l}{ Case study 3: Male or female patients presented by genital ulcer } \\
\cline { 2 - 3 } & Freq. & \% \\
\hline Correct & 7 & 0.70 \\
In correct & 3 & 0.30 \\
Total & 10 & 100 \\
\hline
\end{tabular}

The results showed only $40 \%$ of care providers provide condom to STIs patient, nerve the less they provide condom without counseling or education on how to use it.

Figure three showed that $80 \%$ care providers stated the unavailability of STIs management guide lines and protocols unable them to provide quality STIs health services. Fifty percent stated the poor working environment at PHC canters in El-Damazin as challenges unable them to deliver a quality services.

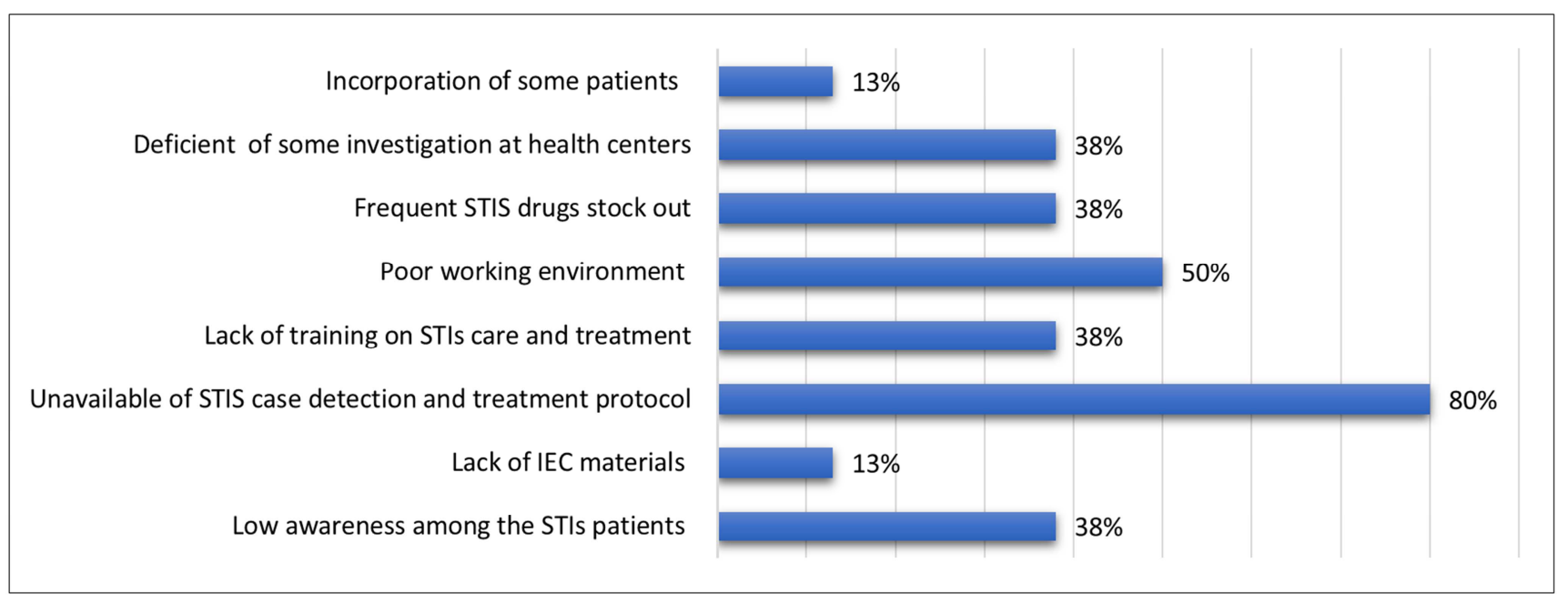

Figure 3. Challenges to provide a quality STIs health services by care providers in the PHC centers at El-Damazin locality in BNS, 2015-2016 ( $n=10)$.

In this paper, table three showed that only three health CPs referred patients for another services during the last three months but, none of them used the referral cards correctly.

Table 3. Patient referral mechanism used by CPs and their knowledge on writing referral card in the eight PHC centers in BNS at El-Damazin locality, 2015$2016(n=10)$.

\begin{tabular}{lllll}
\hline \multirow{2}{*}{ Care providers } & \multicolumn{2}{l}{ Referred any STIs patients' } & \multicolumn{2}{l}{ Those referred STIs patients and used the referral card correctly } \\
\cline { 2 - 5 } & Freq. & $\%$ & Freq. & \% \\
\hline Yes & 3 & 0.30 & 0 & 0.0 \\
No & 7 & 0.70 & 3 & 100 \\
\hline
\end{tabular}

\section{Discussion}

This study assessed both the capacity of PHC centers, and the capacity of health CPs to provide STIs health service that in line with the national standard in El-Damazin locality at BNS during 2015 -2016.
In regards to the PHC centers; In this study the capacity of PHC centers' to provide the STIs service and at the same time keeping up the patient's privacy found unsatisfactory in the most of the studied PHC centers. The findings showed lack of working doors in $25 \%$ of the asseesed consultation rooms. Also, the Patient's records can be reached by other in two third of the studied PHC centers (The findings of this 
study shows only two health centers had a closed cupboard for keeping the patients records away from other that care providers). Also, in this study, the findings revealed that the male to female ration among the STIs patients was 1:1.4 and the high frequency of STIs was among the age group of 25 to 34 years $(53 \%)$. This findings showing the high frequency of the young and the female patients with STIs in the PHC centers at El -Damazin locality in BNS, in such case the perceived value of privacy, confidentiality are critical attributes for efficient STIs services utilization. While WHO stressed on patients confidentiality in several reports $[3,6$, 13, 14]. Addisu. A and Wanzahun. $G$ in their study illustrated, the patients wouldn't prefer to share all information stored with all potential recipients, and the patients with sensitive information preferred less sharing of their data [15], and Sameena et al. mentioned that the patient confidentiality is a critical tenet in all areas of medicine [16].

Although STIs syndromic approach for detection and treatment of STIs was endorsed and adopted for PHC level in Sudan since 2004 [3], yet using STIs syndromic approach wasn't happen in this study due to unavailability of work guidelines and protocols in all studied PHC centers.

Even though the management protocol were unavailable in the PHC centers but the detection of STIs among the patients is high in almost studied PHC centers (One STIs patients every five patients), this result is not in line with literature that shows the effective detection of STIs by using syndromic management approach [14]. Despite the high rate of STIs patients but most of the PHC centers had completed stock of STIs drug supply. The justification, was either STIs were ice purge diseases or STIs drugs may use unregulated or without guidance (misused and overused), it happened in many other developing countries [3-17]. This finding is almost consistent with the result of the study conducted in Pakistan [18] where most providers practiced personal pragmatism that is, following their protocols for treatment with different medications, dosages, and treatment durations. However, this finding is slightly higher than the result of the study conducted in six West African countries, where the competent treatment recommended to $14.1 \%$ of patients in conformity with national syndromic STD management algorithms [19].

Setting up the high-quality STI services is considerably more difficult in resource-poor settings as in El- Damazin locality health centers. The PHC centers had limit resources in term of management protocol, IEC materials, and medical equipment. This status similar to the sub Saharan countries, where R. G. White found a huge gap in resources and recommended that the health policy requires that scarce resources to be allocated to interventions with the best costeffectiveness even if relative impact at the population level is modest [20]. Also, Peterman et al. highlighted that there is need to develop and to adopt more tools (such as intervention toolkits, checklists, and implementation guides) that help program staff in the provision of quality services. Therefore, availability of standard equipment and supplies seeks to ensure that all components of an evidence-based package of services are delivered at the facilities in a quality manner and according to the level of care as per national protocols and guidelines [21].

In regards to the health $\mathrm{CPs}$; The inequitable gender distribution among the health care providers was found in this study (The majority of care providers are male), the majority with less than two years' experience, this may increase the culture of silence about STIs especially this study the PHC centers records illustrated more than half of the STIs patients are female. Associating the three results together with the result that showed more than third of care providers $(38 \%)$ had a challenges due the low awareness among the STIs patients indicates that care providers needs more skills to provide a proper education and counseling to STIs patients and to communicate or discuss the sensitive STIs issues like the condom or practicing unsafe sex especially with female patients.

This study revealed all care providers treat the STIs patients without guidance and they depend on their work experience on top of their health background. The national protocol for STIs care and treatment indicates; each care provider should follow and use the national STI syndromic management guidelines correctly [6-7], this means care providers should have adequate training on syndromic management of STIs, but in this study, the majority of care providers didn't receive in-service training programs for three years (only 20\% of care providers received in service training on syndromic management approach) and more than one third of care providers perceived the lack of training is a challenge on providing quality STIs service.

This research is similar to a survey conducted in Windhoek, Namibia among private general practitioners where none of them had specific training on syndromic management [22], and different from the result of South Africa survey [23], which showed that South Africa managed the diversity of knowledge found among investigated care providers in country by standardizing the STIs service provision through the continuous capacity building of all the staff involved in STIs service delivery. In this study, even the overall knowledge of care providers varied by syndrome type, but it was all lowest $(70 \%$ of care providers incorrect diagnosed the urethral discharge case study, 60\% incorrect diagnosed the vaginal discharge case study and $30 \%$ incorrect diagnosed the genital ulcer case study). This result is lower than the result of the KAP survey conducted on general practitioners in Karachi, Pakistan [24], and among private general practitioners in Windhoek, Namibia, [22] where the knowledge for urethral discharge, e.g., was, $55.3 \%$ and $56.5 \%$, this might be because these studies only focused on knowledge of general practitioners while our study included lower level health workers rather than general practitioners (medical doctors).

This study revealed that all care providers who referred STIs patients during the last three months didn't used the referral card correctly. More than one third of care providers not oriented with STIs syndromic management approach standard requirements $38 \%$ of care providers perceived the 
deficit of some investigation materials is a challenge facing the implementation of the national guideline for STIs care and treatment). Association of the two findings indicating rather than care providers training and avail the needed resources, they need to build their capacities in different required skills for service provision (e.g. like referral mechanism, communication skills, reporting and archiving the health documents) to enable them to perform their duties more efficiently [3-4]. Also, V. Verhoeven et al study; recommend to engage the care providers in useful education and communication programs activities to build their capacities towards improving treatment-seeking behavior among the STIs patients [25].

\section{Conclusion}

This study investigated the PHC centers and health care providers regarding the factors affecting the utilization of STIs health services. The general observation in this study that the implementation of the STIs management activities by the PHC centers in El-Damazin locality doesn't satisfactorily meet the recommended national guidelines for STIs management and several factors explored at the level of PHC centers might acted as barriers to the provision of quality services. (E.g. although all PHC centers had separated private medical consultation room; nevertheless, keeping up the patient's privacy found unsatisfactory in the most of the studied PHC centers). Most of the facilities had completed quota of drug supply. The PHC centers faced unavailability of management protocol, IEC materials, and additionally unavailability of completed equipment. Concerning the care providers; most of them were Male, medical doctors, had minimal years experiences, didn't received in-service training programs, they had discrepancy knowledge regarding STIs diseases and they faced several problems to provide a quality service to STIs patient. The inequitable gender distribution among the CPs may increase of silence about STIs especially more than half of STIs patients are female.

\section{Recommendations}

The study findings revealed some factors affecting the utilization of Sexual Transmitting Infections Health Services. The following are therefore recommended based on the results.

1- For the confidentiality issue related to service provision, patients with diagnosed STIs should be treated safely and appropriately across the national guidelines; it's essential to sensitize the care providers' to archive the patient's files in secure place and to improve the condition of the medical consultation rooms at the PHCs.

2- Avail the needed medical equipment in PHC centers to enable the provision of comprehensive STIs services that is in line with national standard (e.g. avail drug supply that in line with national protocol for STIs care and treatment, STIs management protocol, IEC materials, and avail completed equipment at each facility).

3- Investment on health care providers to improve quality of STIs service provision by continuous in-service training on syndromic management of STIs, and to engage them in other capacity building programs.

4- In line to develop comprehensive strategic intervention, this study recommend to conduct another study in same state and in other states to enrich the evidences on the factors affecting the utilization of the STIs services at the demand side.

\section{References}

[1] Christian N, Janet B: What are sexually transmitted infections? What are sexually transmitted disease?. Medical News Today; 2012 Jun 13 [Updated 2017 July 26].

[2] Newman L, Rowley J, Vander Hoorn S, Wijesooriya NS, Unemo M, Low N, Stevens G, Gottlieb S, Kiarie J and Temmerman M: Global Estimates of the Prevalence and Incidence of Four Curable Sexually Transmitted Infections in 2012 Based on Systematic Review and Global Reporting. PLoS One. 2015 Dec 8; 10 (12):e0123304.

[3] WHO. Global Health Sector Strategy on Sexually Transmitted Infections 2016-2021. June 2016. Page, 12.

[4] WHO. Guidelines for the Management of Sexually Transmitted Infections. Essential Medicines and Health Products Information Portal. WHO:2001, page 88, Updated 2006.

[5] UNAIDS-Sudan. Global AIDS Response Progress Reporting $2012-2013$.

[6] Sudan. National AIDS Control Programme Report on ANC: HIV Sentinel Sero-Survey 2009. Khartoum: SNAP surveillance unit. 2010.

[7] WHO, Global prevalence of selected available sexually transmitted infections. WHO; 2001 Nov.

[8] UNAIDS. 2010 UNGASS Report, North Sudan [internet]. SNAP; 2010 March 31.

[9] FMOH: The National Strategy for Reproductive Health 20062010. page12.

[10] FMOH- SNAP: Distributed in the 15 northern States [Internet]. page 10; 2010.

[11] WHO, Programme areas, WHO's interventions in Blue Nile. WHO EMRO; 2015 Aug 7 [updated 2015Feb 12].

[12] Sudan. National AIDS Control Programme Report on ANC: HIV Sentinel Sero-Survey 2009. Khartoum: SNAP surveillance unit. 2012.

[13] World Health Organization, 10 Facts on Sexually Transmitted Infections, World Health Organization, Geneva, Switzerland, 2013.

[14] World Health Organization and National AIDS Commission. Management of Sexually Transmitted Infections Using Syndromic Management Approach; Guidelines for Service Providers. Ministry of Health, Lilongwe, Malawi, 3rd edition, 2007. 
[15] Addisu. A, Wanzahun. G. Knowledge and Practice of Clinicians regarding Syndromic Management of Sexually Transmitted Infections in Public Health Facilities of Gamo Gofa Zone, South Ethiopia. Journal of Sexually Transmitted Diseases. Volume 2015 (2015), Article ID 310409, 6 pages.

[16] Sameena Kausar, Caroline S Bradbeer. Risk management Patient confidentiality in STIs: current guidance and legal issues. The Obstetrician \& Gynecologists. 240 Risk management 2006; 8:240-244. 10.1576/toag.8.4.240.27274.

[17] Ali. M. M. Factors influencing healthcare service quality. International journal of health policy and management. Received 2012 Apr 26. Accepted 2012 Jul 21.

[18] World Health Organization, 10 Facts on Sexually Transmitted Infections, World Health Organization, Geneva, Switzerland, 2013.

[19] R. Bitera, M. Alary, B. Mâsse et al., "Quality of disease management of sexually transmitted diseases: investigation of care in six countries in West Africa," Cahiers Sante, vol. 12, no. 2, pp. 233-239, 2009.

[20] White. R. G. "Curable sexually transmitted infection treatment interventions to prevent HIV transmission in Sub-Saharan Africa," The Open Infectious Diseases Journal, vol. 3, no. 1, pp. 128-155, 2009.
[21] Peterman. A. Effective Interventions to Reduce Sexually Transmitted Disease: Introduction to the Special Issue. Sexually Transmitted Diseases: February 2016 - Volume 43 Issue 2S - p S1-S2. doi: 10.1097/OLQ.0000000000000373. Editorial.

[22] S. N. Iipinge and L. Pretorius, "The delivery and quality of sexually transmitted infections treatment by private general practitioners in Windhoek Namibia," Global Journal of Health Science, vol. 4, no. 5, pp. 156-171, 2012. View at Google Scholar View at Scopus.

[23] Zanakis. SH, Alvarez. C, Li V. Socio-economic determinants of HIV/AIDS pandemic and nation's efficiencies. European Journal for Operational Research, 2007; 176:1811-38. Received 26 August 2004; accepted 3 October 2005.

[24] Hussain. MF1, Khanani. MR, Siddiqui. SE, Manzar. N, Raza. S, Qamar SM. F. A. Knowledge, attitudes \& practices (KAP) of general practitioners (GPs) regarding sexually transmitted diseases (STDs) and HIV/ AIDS in Karachi, Pakistan. Journal of the Pakistan Medical Association, vol. 61, no. 2, pp. 202205, 2011.

[25] FHI - Control of Sexually Transmitted Diseases, A Handbook for the Design and Management of Programs. 